\title{
Wavelength discrimination at detection threshold
}

\author{
K. T. Mullen \\ Physiological Laboratory, Downing Street, Cambridge CB3 3EG, UK
}

\section{J. J. Kulikowski}

Visual Sciences Laboratory, The University of Manchester Institute of Science and Technology, P.O. Box 88, Sackville Street, Manchester M60 1QD, UK

Received November 28, 1988; accepted October 24, 1989

\begin{abstract}
The experiments that we report aim to elucidate the linkage between cone outputs and color sensation. This is investigated by measuring wavelength discrimination between stimuli at threshold levels of detection. Stimuli are large spots $(0.75 \mathrm{deg})$ presented on a white background. A $2 \times 2$ alternate forced choice method is used to measure simultaneously the detection of different wavelengths and discrimination between them. This method reveals at least four distinguishable colors, indicating the presence of four different sets of mechanisms at threshold. These are associated with the color sensations of orange, pale yellow, green, and blue. There is also evidence for a fifth imperfectly distinguished color (violet) in the shortest wavelength region. Results show that the boundaries between the distinguishable colors have little variation in their spectral positions. This is compatible with the presence of fixed perceptual boundaries in the spectrum dividing the different types of detection mechanism. The correspondence of the spectral locations of the distinguishable colors to the cone opponent responses revealed in the spectral sensitivity function suggests that these color sensations are postreceptoral in origin, arising from different combinations of the three cone outputs.
\end{abstract}

\section{INTRODUCTION}

Individually, each of the three types of cone in the human retina is able to signal information about the number of absorbed quanta but not their wavelength. Thus it is the postreceptoral combination of these cone outputs into different neural channels that is the first step in enabling a wide range of colors to be distinguished. Both neurophysiological and psychophysical evidence suggests that the initial stage of this postreceptoral processing involves the inhibitory, linear combination of the cone outputs into cone opponent channels. However, the relationship linking the original cone activities and the cone opponent responses to the sensation of color remains a complex problem that requires elucidation.

In these experiments we test for the presence of chromatically tuned mechanisms associated with different color sensations across the spectrum. The minimum number of distinct color sensations across the spectrum at threshold is identified by measuring simultaneously the detection of different wavelengths and discrimination between them in a forced choice procedure. The results may be used to indicate a minimum number of neural mechanisms involved in the detection of the spectrum and their respective bandwidths. Similar methods, which are discussed further below, have been used previously in the identification of spatial frequency, temporal frequency, and orientation selective mechanisms in vision. ${ }^{1-6}$ Similar methods have also been applied to wavelength discrimination for a variety of different purposes, and the results indicate that under certain conditions some wavelengths can be distinguished at threshold. ${ }^{7-10}$ Thus, while these results indicate that more than one mechanism is potentially active in the detection of the spectrum, a systematic, step-by-step identification of the number of different color sensations across the whole spectrum has not been attempted.

A method of color discrimination at detection threshold has also been used previously in order to test for the presence of particular chromatic mechanisms within the color space, ${ }^{11}$ and the results are compatible with the presence of multiple, higher-order mechanisms. However, discrimination was measured only between preselected points in color space, and a systematic spanning of the space required to reveal more completely the number of distinguishable colors at threshold was not made. Thus it remains unresolved whether wavelength discrimination across the spectrum at threshold would reveal detection by lower-order mechanisms, such as the cone opponent ones, or whether detection would be by higher-order mechanisms more narrowly tuned to wavelength, for example, of the type found neurophysiologically in primate prestriate cortex. ${ }^{12}$

A further aim of the present experiments is to determine whether the boundaries between distinct colors in the spectrum occur at fixed or variable spectral locations. Fixed transitions in the spectrum would indicate the presence of perceptual boundaries between distinct categories of spectral sensation and would be compatible with the coding of the spectrum by discrete neural mechanisms. Alternative$l y$, mutable boundaries would indicate that wavelength discrimination at threshold depends solely on the difference in wavelength between stimuli, with no categorical performance limits.

There is evidence for two categorical color sensations, arising respectively from the stimulation of a single mediumwavelength (M) or long-wavelength $(\mathrm{L})$ cone. ${ }^{13,14}$ The question with which we are concerned is whether such discrete sensations arise from the stimulation of postreceptoral chromatic mechanisms, when more than one type of cone and 
much larger numbers of receptors are likely to be active. For this reason, relatively large test stimuli (0.75-deg spots) of long duration (500 msec) are used since these spatiotemporal conditions are known to be favorable to color detection. ${ }^{15-18}$ To obtain photopic conditions, the stimuli are presented to a uniform white background of $1000 \mathrm{Td} .{ }^{15,19}$

Measurements of discrimination at a fixed level of detection are usually interpreted by making certain assumptions about the detection process. First, it is generally assumed that the response of the detecting mechanism is univariant. Threshold models assume that a single neural mechanism can determine threshold detection (whichever is the most sensitive) and that this forms a labeled line. Integration of responses across different mechanisms is determined by probability summation among them. Perfect discrimination among stimuli requires that each be detected by a different, independent mechanism, whereas failure to discriminate among stimuli indicates that they are both detected by the same mechanism. Continuous models, on the other hand, consider that there is no threshold and that the response to a stimulus is determined by a weighted sum of a range of active neural mechanisms. Discrimination will depend on the bandwidths and number of active mechanisms, and perfect discrimination will occur when stimuli are at least a bandwidth apart. In practice it is often difficult to decide from experimental data which model is the most applicable. For further discussion see Refs. 3-5 and 7 .

Although these models provide different frameworks for the interpretation of the results, they are by no means a requirement for the experimental determination of the number and the location of distinguishable colors in the spectrum at equivalent levels of detectability. Furthermore, both types of model agree that perfect discrimination between stimuli indicates detection by independent mechanisms, which allows the number and location of chromatic mechanisms to be determined without reference to one particular model. In this paper, results are reported within the framework of the threshold model used by Watson and Robson. ${ }^{4}$ However, the interpretation of the results may be made in other ways, and these are raised in the Discussion section below.

Some preliminary results were reported at a meeting of the Northern Eye Institute. ${ }^{20}$

\section{METHODS}

\section{The Stimulus}

The stimulus is a circular test spot of variable wavelength, with a $0.75-\mathrm{deg}$ diameter and of $500-\mathrm{msec}$ duration. It is centrally fixated by using a surrounding group of four small fixation marks. The test spot is superimposed upon a circular, uniform white background, $8 \mathrm{deg}$ in diameter and at 2500 $\mathrm{K}$. The background intensity is $1000 \mathrm{Td}$ unless given otherwise.

\section{Apparatus and Calibrations}

A two-channel Maxwellian-view system is used as shown in Fig. 1. A Czerny-Turner grating monochromator of 5.7-nm bandwidth is used to produce the test spots. The intensity of the test spot and background is varied with a calibrated 4ND circular wedge. All light sources are 50-W quartz-halo-

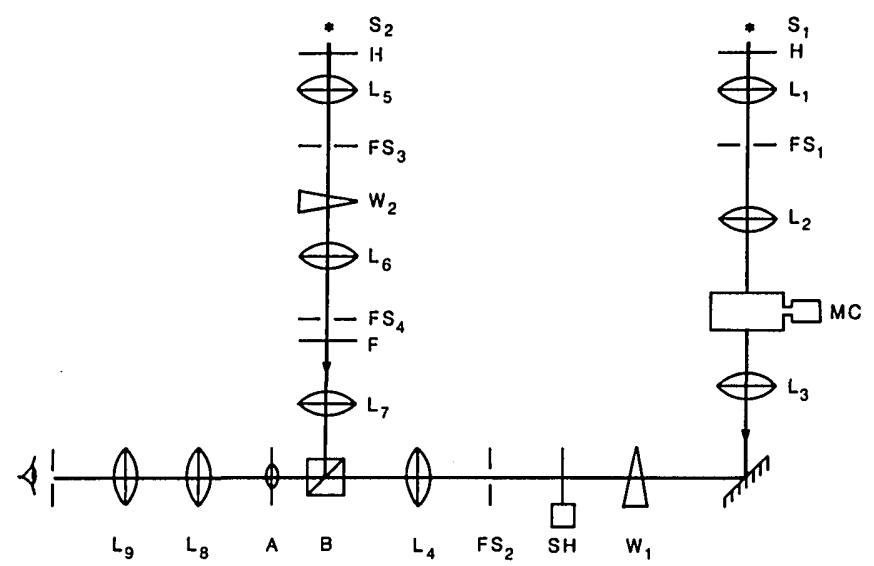

Fig. 1. Two-channel Maxwellian-view system. A, achromatizing lens; B, beam splitter; F, fixation mark; FS's, field stops; H's, heat filters; L's, lenses; MC, monochromator; S's, sources; SH, shutter; W's, neutral-density wedges.

gen bulbs slightly underrun at $11.4 \mathrm{~V}$. A shutter is used to control the duration of the stimulus. An achromatizing doublet is used to correct axial chromatic aberrations.

The system was calibrated with a photomultiplier with an S cathode (P-M 733S-272), and subsequently absolute intensities were measured using a calibrated United Distributor Technology radiometer with a P-I-N photodiode. The luminance of the white background was regularly calibrated with a Photo Research (PR 1500) spot photometer with an internal calibration source. The monochromator was calibrated from the sharp absorbance peaks of a holmium filter. The subjects are the authors, and both perform normally on all color vision tests including the Farnsworth Munsell 100 hue test and on the Nagel anomaloscope.

\section{Procedure}

The overall aim of the procedure is to measure wavelength discrimination between test stimuli at equivalent and threshold levels of detectability. A $2 \times 2$ alternative forced choice (AFC) procedure is used to measure simultaneously the detection of and discrimination between two stimuli of different wavelengths. The method closely resembles that of Watson and Robson. ${ }^{4}$ A trial consists of two intervals in time. One is blank, containing only the white background, and in the other the spot stimulus is presented superimposed upon the background. The spot is randomly one of two wavelengths $\left(\lambda_{1}\right.$ or $\left.\lambda_{2}\right)$. The subject indicates in which interval the stimulus appeared and also whether it was $\lambda_{1}$ or $\lambda_{2}$. Within a single experiment, each wavelength is presented at least 50 times at each of 4 or 5 randomly interleaved, different intensities spanning threshold. Hence each measure of discrimination at detection threshold is based on 400-500 trials.

The random interleaving of the different intensity levels avoids cues to the discrimination between stimuli being gained from any small differences in the levels of the detectability of the two wavelengths. As a further precaution the intensity levels at which each stimulus was presented were selected to span equivalent ranges of their psychometric functions. This was done by using a method of adjustment as an approximate measure of threshold and then using trial runs of the $2 \times 2 \mathrm{AFC}$ procedure to select the intensity levels 
for the two stimuli. This procedure also served to allow the subject to become thoroughly familiar with the appearance of the stimuli pairs at low levels of detectability before beginning the experiment.

For each wavelength pair the detection of each of the two stimuli as a function of intensity is obtained and also measurements of the discrimination of one stimulus from the other. Results for two different wavelength pairs are shown in Fig. 2 and have been selected to illustrate the range of results that may be obtained. For a wavelength pair of 530 with $610 \mathrm{~nm}$, shown in the top two panels, the results for detection (filled circles) and discrimination (crosses) lie very close together, indicating that these stimuli can be distinguished as well as they can be detected. For a different wavelength pair of 610 with $655 \mathrm{~nm}$ (lower two panels), the results for detection and discrimination are well separated. Discrimination is at or close to $50 \%$ as detection levels rise.

\section{Analysis of Results}

The analysis of the results requires that some measure of the separation between detection and discrimination be obtained. The data from the $2 \times 2 \mathrm{AFC}$ experiment (four psychometric functions) were transformed with a probit analysis, and best-fitting linear regressions were fitted. The separation between detection and discrimination functions along the performance axis is measured by comparing discrimination at a single level of detectability. A detection level of $90 \%$ correct is selected, and this value is taken as detection threshold. At $90 \%$ detection, discrimination may range from chance (50\%) to perfect $(90 \%)$ when it is as good as detection. In the figures, these values for discrimination are plotted as relative values ranging from 0 for chance to 1 for perfect. The two values for discrimination obtained from each $2 \times 2$ AFC procedure are averaged to eliminate any response bias to one of the stimuli. The assumption
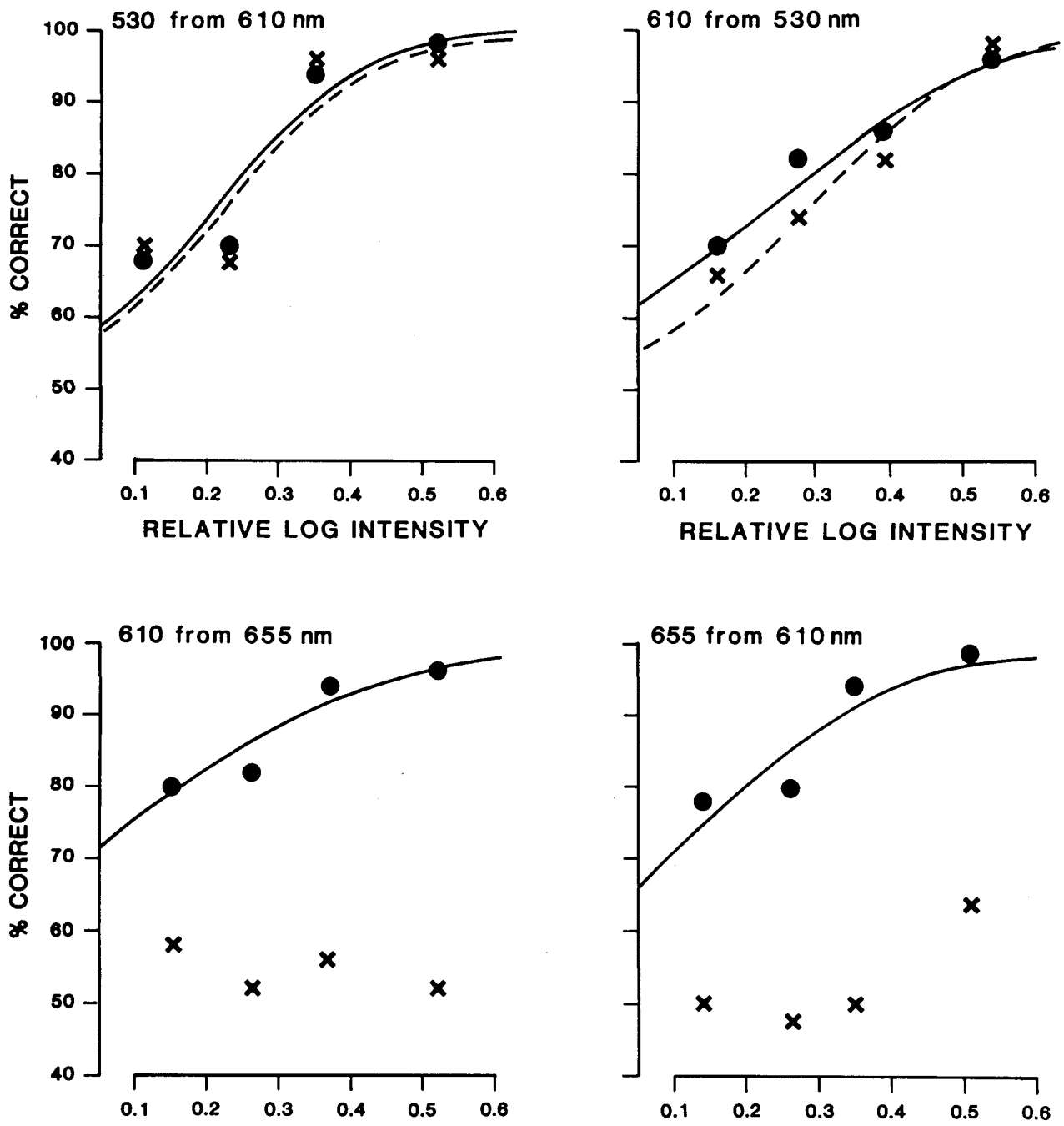

RELATIVE LOG INTENSITY

RELATIVE LOG INTENSITY

Fig. 2. Two sets of results obtained from an AFC procedure, one using wavelength pairs of 530 with $610 \mathrm{~nm}$ (upper panels) and the other 610 with $655 \mathrm{~nm}$ (lower panels). Percentage correct is plotted as a function of the relative intensity of the stimulus in log units. The solid curves with filled circles indicate the psychometric function for detection of the stimulus, and the dashed curves with crosses show the function for the discrimination of one stimulus from the other. All curves are fitted by using a probit analysis. Detection threshold is taken as the stimulus intensity that is $90 \%$ correctly detected, and at this intensity the percentage correctly discriminated is calculated. Percent correctly discriminated may range from chance (50\%) to perfect $(90 \%)$ and is expressed as a relative value from 0 to 1 , respectively (see text). (Subject KTM.) 
underlying the analysis of the data is that a probit analysis provides a reasonable linear fit to the data, and this is supported by the results.

\section{RESULTS}

The first aim of the experiments is to determine the minimum number of the distinguishable sensations across the spectrum for stimuli at detection threshold (a detectability of $90 \%$ ). In a series of $2 \times 2 \mathrm{AFC}$ experiments, the difference in wavelength between the two stimuli is increased by holding one wavelength fixed and altering the other wavelength in each successive AFC experiment. The wavelength separation is increased until discrimination is as good as detection, indicating the presence of two perfectly distinguishable colors. Another fixed wavelength is selected within the newly identified perfectly distinguishable region, and the process is repeated. This method is used to count the minimum number of perfectly distinguished colors across the spectrum and hence the number of independent mechanisms determining detection.

Results for subject KTM are shown in Fig. 3 and those for JJK in Fig. 4. For each set of wavelength pairs, the wavelength that remains fixed is given by the filled symbol, and the other member of the pair in each successive experiment is given by the open symbol. Hence the first set of results (Figs. 3a and 4a) is for a fixed wavelength of $520 \mathrm{~nm}$ paired with longer wavelengths. For both subjects, once the paired wavelength is $\sim 575 \mathrm{~nm}$ or longer, relative discrimination is very close to unity, indicating that the two wavelengths can be distinguished from each other as well as they can be detected.

It now becomes useful to define a point that can be taken to mark the division between distinguishable spectral regions. A relative discrimination of unity indicates that stimuli are perfectly distinguishable and is the best theoretical measure of whether stimuli are detected by independent mechanisms. The disadvantage of using this criterion in practice, however, is that it is difficult to estimate accurately. Hence as a working measure a lower criterion has been selected of a relative discrimination of 0.5 . This is the midpoint on a psychometric function that describes discrimination at a fixed level of detection as a function of wavelength, and it corresponds to a discrimination of $70 \%$ at the detection level of $90 \%$. A different choice of detection level as threshold will not affect the location of the boundaries between distinguishable colors provided that the same relative point on the psychometric function is used. Furthermore as the selection of the next fixed wavelength requires that perfect (or near perfect) discrimination is achieved, the choice of a criterion below unity in a definition of the boundary between distinguishable colors does not affect the count of the number of separable mechanisms but simply permits a more precise location of the boundaries between them. In Figs. $3 \mathrm{a}$ and $4 \mathrm{a}$ the boundary between distinguishable colors occurs at $566 \mathrm{~nm}$ and shows that a wavelength separation of $46 \mathrm{~nm}$ is required to reach a relative discrimination of 0.5 .

The next fixed wavelength selected is $575 \mathrm{~nm}$. This wavelength is chosen because it lies within the second distinguishable region of the spectrum and at a point where relative discrimination has reached asymptotic levels at or close to unity. The results are shown in Figs. $3 \mathrm{~b}$ and $4 \mathrm{~b}$ (righthand sides). Once the paired wavelength is $\sim 595 \mathrm{~nm}$ or longer, the two stimuli can be perfectly discriminated at threshold, and hence the presence of a third distinguishable region in the spectrum is revealed. The boundary dividing

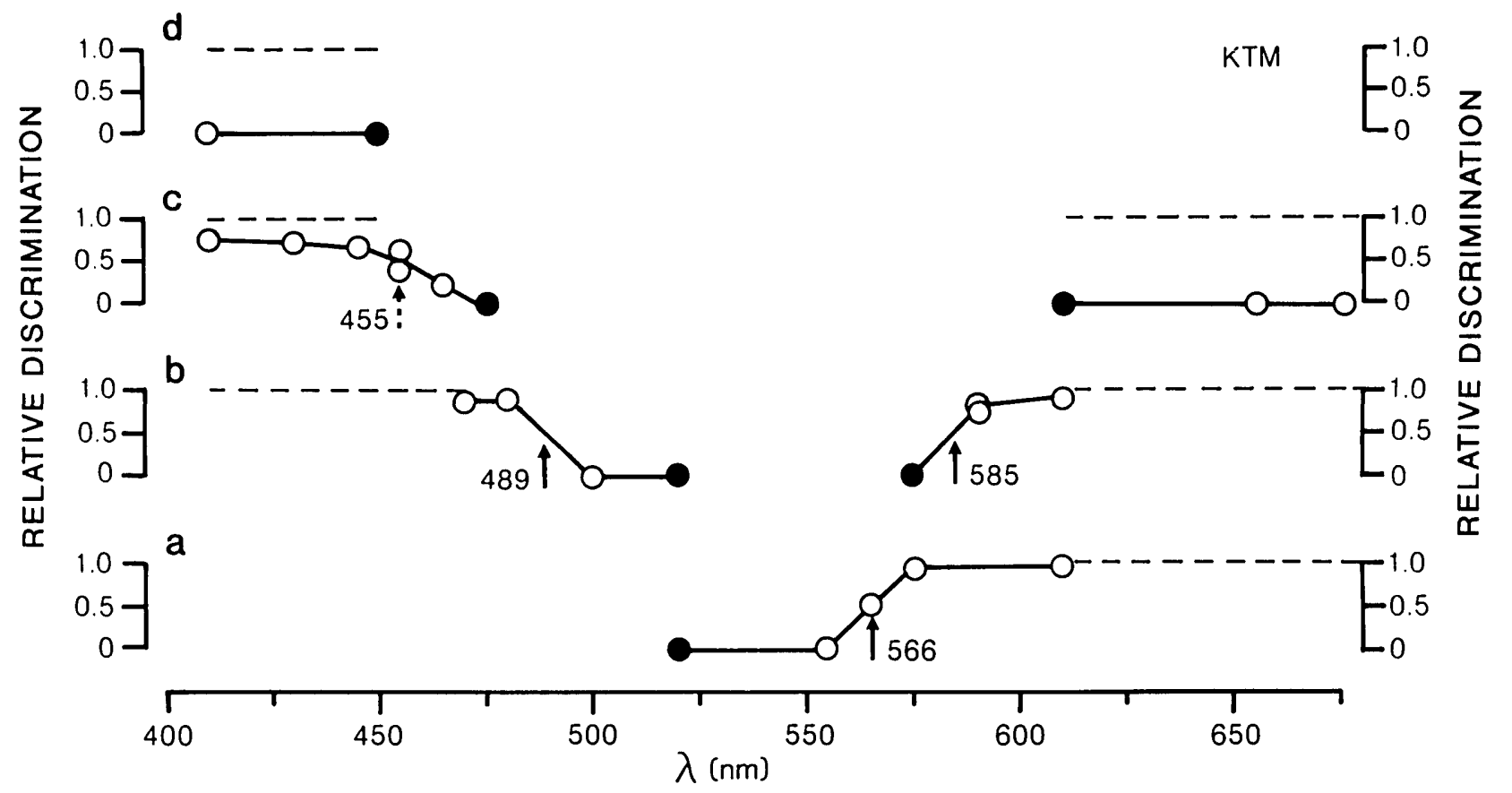

Fig. 3. Correct discrimination at detection threshold (90\% correct detection) is plotted in relative units for each pair of wavelengths. In each series of pairs, one wavelength remains fixed (filled circles), and the other member of the pair is indicated by open circles. Dashed lines indicate perfect discrimination (unity). Vertical arrows and numbers show the wavelength at which relative discrimination from the fixed wavelength is 0.5 and marks the division between perfectly distinguishable spectral regions. (Subject KTM.) 


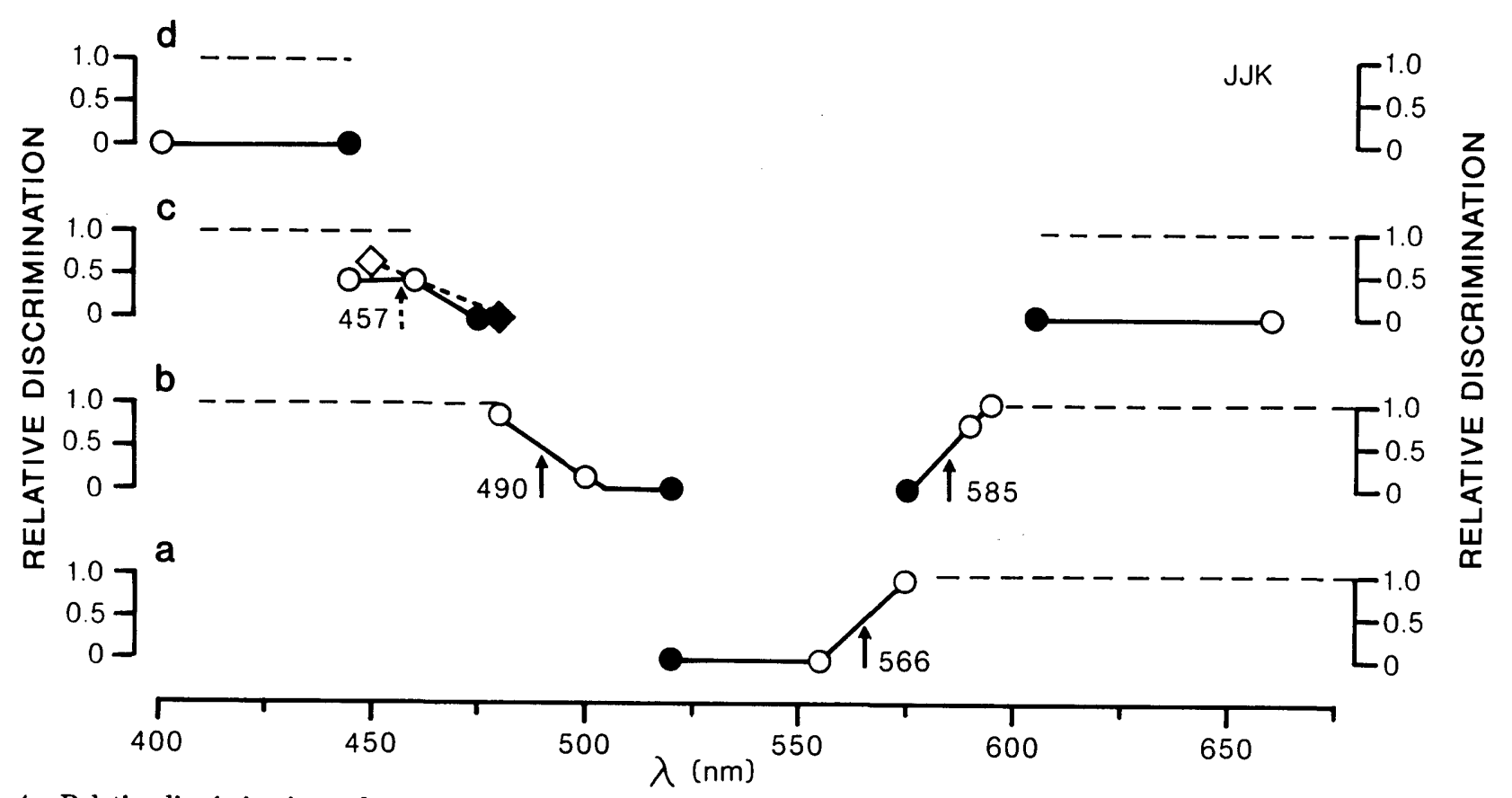

Fig. 4. Relative discrimination at detection threshold (90\% correct detection) is plotted for each pair of wavelengths as Fig. 3. (Subject JJK.)

these second and third regions is the same for each subject and lies at $585 \mathrm{~nm}$. The next fixed wavelength is $610 \mathrm{~nm}$ (right-hand sides of Figs. 3c and 4c). The results show that the discrimination of all longer wavelengths from this at threshold remains at chance levels. Thus overall the results shown indicate the presence of three distinct colors in the spectrum above $520 \mathrm{~nm}$.

The same approach is used to measure discrimination at shorter wavelengths below $520 \mathrm{~nm}$, and the results are shown in Figs. $3 \mathrm{~b}$ and $4 \mathrm{~b}$ (left-hand side). These figures show that there is a fourth distinguishable color with a boundary at $489 \mathrm{~nm}$ for KTM and $490 \mathrm{~nm}$ for JJK.

There is also evidence for a fifth distinguishable region at shorter wavelengths (Figs. $3 \mathrm{c}$ and $4 \mathrm{c}$, left-hand side). Discrimination of shorter wavelengths from the fixed wavelength of $475 \mathrm{~nm}$ is elevated above chance levels but remains poorer than in the other regions. Discrimination in this region at threshold remains considerably below perfect despite increases in the wavelength interval and regardless of fine adjustments of the location of the fixed wavelength. For subject KTM, altering the fixed wavelength by $5 \mathrm{~nm}$, from 475 to $480 \mathrm{~nm}$, fails to improve discrimination. For JJK, discrimination of shorter wavelengths from $475 \mathrm{~nm}$ is very poor. Altering the fixed wavelength to $480 \mathrm{~nm}$ produces a slightly improved discrimination that allows a boundary to be set at $457 \mathrm{~nm}$ (shown in Fig. $4 \mathrm{c}$ with diamond symbols and dashes). These results suggest that there may be a fifth distinguishable color for stimuli presented above detection threshold. Finally., the results of Figs. $3 \mathrm{~d}$ and $4 \mathrm{~d}$ show that no shorter wavelengths can be distinguished from $450 \mathrm{~nm}$ at threshold.

The results for the two subjects indicate that there are at least four perfectly distinguishable color sensations at threshold across the spectrum. Both subjects agree that the appearance of these colors at threshold, in order of decreas- ing wavelength, is orange, pale yellow, green, and blue. The fifth poorly discriminated, color in the short-wavelength region appears violet at threshold.

\section{Test for the Constancy of the Boundary Locations}

The next experiments address the question of whether each of the boundaries dividing the different color sensations across the spectrum is mutable or fixed. If discrimination depends solely on the difference in wavelength between stimuli, the spectral location of the boundary dividing the perfectly distinguishable sensations will shift in response to a change in the location of the fixed wavelength. On the other hand, the boundaries between the distinguishable colors may be fixed in their spectral location, and discrimination between two stimuli requires that each lie on opposite sides of a perceptual boundary. These two possibilities have different implications for the neural processing of wavelength, which are raised in the Discussion section.

In the next experiments the fixed wavelengths are systematically varied, and the positions of the three boundaries dividing the four different color sensations are measured. The results for discrimination between the first and the second color sensation (termed green and pale yellow) are given for both subjects in Fig. 5. Three different fixed wavelengths are used: 520,530 , and $550 \mathrm{~nm}$, shown in the lower, middle, and upper sections, respectively. (The results for the fixed wavelength of $520 \mathrm{~nm}$ have already been shown in the previous figures but are added here to permit comparison.) The discrimination of longer wavelengths from each of these fixed wavelengths $(520,530$, and $550 \mathrm{~nm})$ reaches a relative discrimination of 0.5 at 566,566 , and 569 $\mathrm{nm}$, respectively, for KTM and 566, 566, and $565 \mathrm{~nm}$ for JJK. Thus the division between these two distinguishable regions of the spectrum are coincidental to within $1 \mathrm{~nm}$ for JJK and $3 \mathrm{~nm}$ for KTM despite a $30-\mathrm{nm}$ difference in the fixed wave- 
length. This suggests that discrimination between these two colors is not simply determined by the size of the wavelength difference between stimuli.

In the next experiment a test is made of whether this property also applies to the boundary between the second and third distinguishable colors (termed pale yellow and orange). The pale yellow region is narrow, and so little variation of the fixed wavelength within this spectral region is possible. Instead, different fixed wavelengths within the third distinguishable (orange) region are selected, and discrimination of these from shorter wavelengths is measured. Results are given in Fig. 6 for both subjects. (The results for the original measurements of these two distinguishable regions are included in the lower section of the figure.)

For KTM, fixed wavelengths of 575,610 , and $630 \mathrm{~nm}$ produce boundaries among distinguishable sensations at
585,588 , and $586 \mathrm{~nm}$, respectively. For JJK, the boundary positions are invariant (at $585 \mathrm{~nm}$ ) for the two fixed wavelengths of 575 and $610 \mathrm{~nm}$. Hence the boundary between distinguishable sensations in one subject varies by $3 \mathrm{~nm}$, although not in a direction consistent with a systematic shift in boundary location. Thus the division between the second and third distinguishable regions also shows little variation in its spectral location.

In the same manner, the location of the division between the green and blue distinguishable regions is measured for different fixed wavelengths, and the results for both subjects are given in Fig. 7. Discrimination of shorter wavelengths from three fixed wavelengths is shown for each subject (530, 520 , and $510 \mathrm{~nm})$. For KTM, the divisions among distinguishable colors occur at 488,488 , and $485 \mathrm{~nm}$, respectively, and for JJK, they lie at 490,490 , and $488 \mathrm{~nm}$. For the $20-\mathrm{nm}$
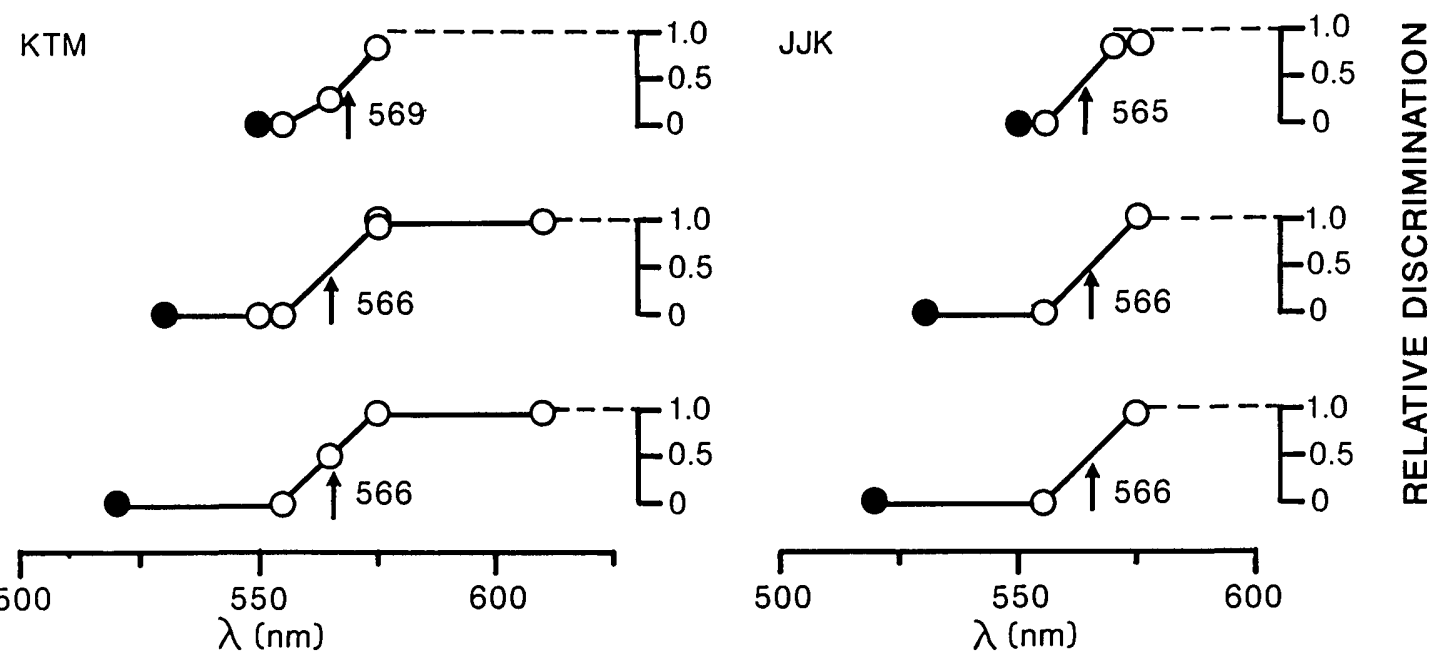

Fig. 5. Relative discrimination at detection threshold is plotted as for Figs. 3 and 4 for KTM (left-hand panel) and JJK (right-hand panel). Results are for discrimination of longer wavelengths from three fixed wavelengths (520,530, and $550 \mathrm{~nm}$ ). Vertical arrows and numbers indicate the position of the boundaries dividing distinguishable spectral regions. The two spectral regions are green and pale yellow in appearance.
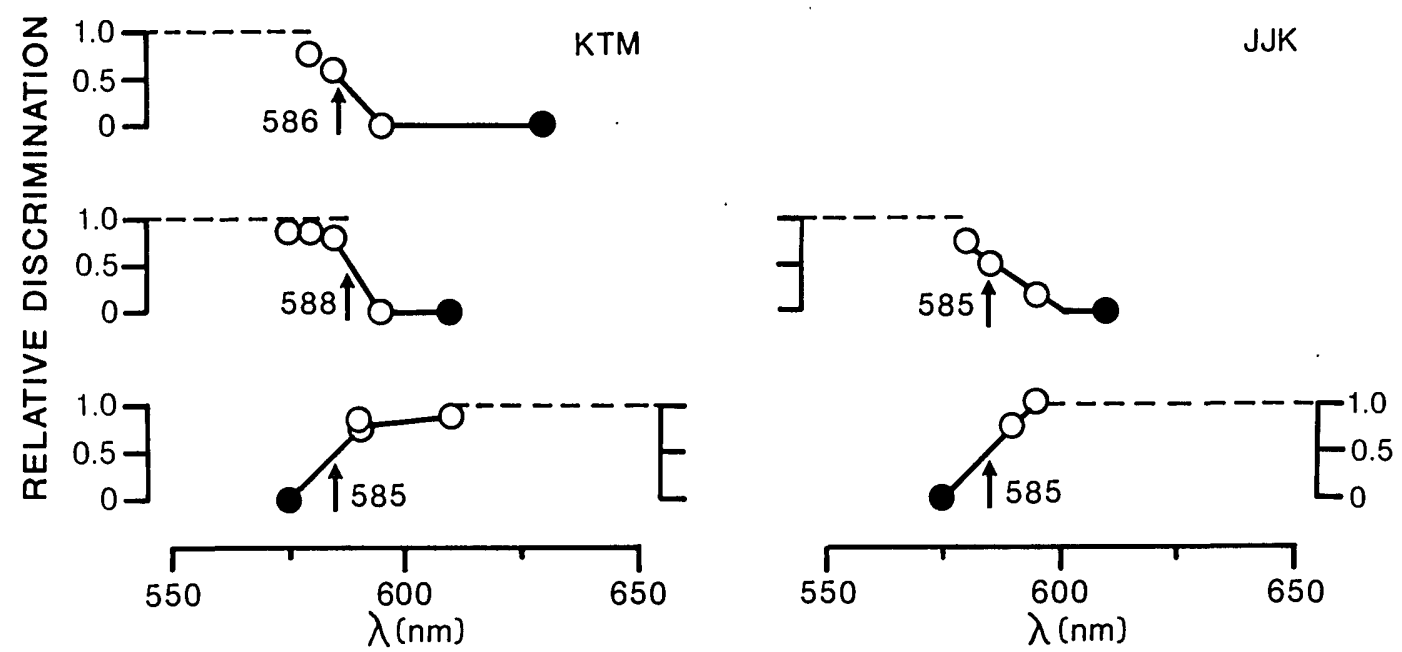

Fig. 6. Relative discrimination at detection threshold is plotted as for Figs. 3-5. Results for ITTM (left-hand panel) show discrimination from three fixed wavelengths $(575,610$, and $630 \mathrm{~nm})$, and results for JJK (right-hand panel) are for two fixed wavelengths $(575 \mathrm{and} 610 \mathrm{~nm})$. The positions of the vertical arrows indicate the division between distinguishable spectral regions. The two spectral regions are pale yelow and orange in appearance. 

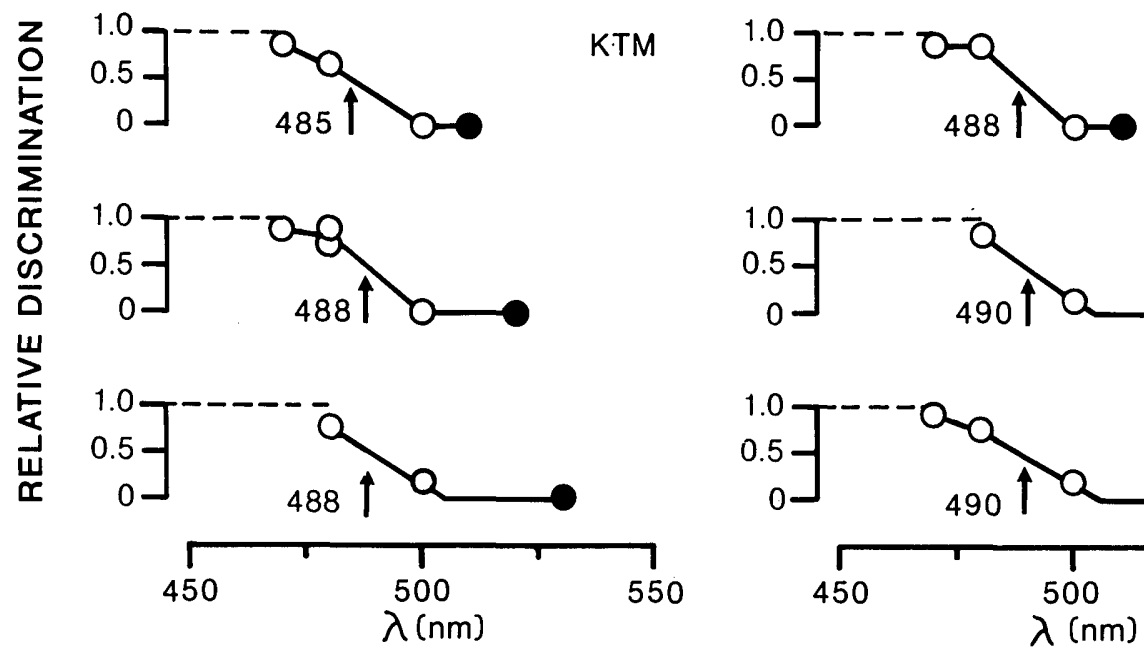

JJK
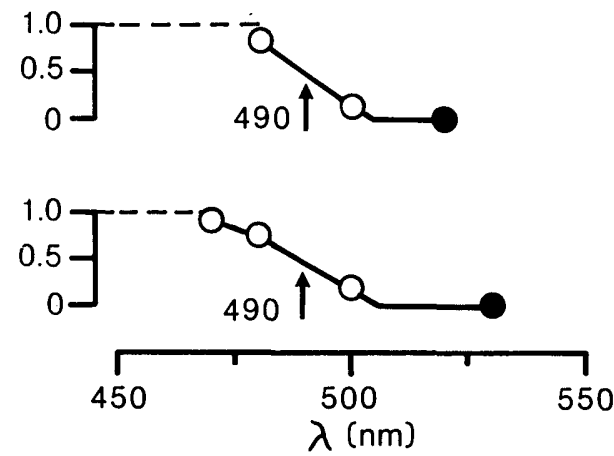

Fig. 7. Relative discrimination at detection threshold is plotted as for Figs. 3-6. Results are for the discrimination of shorter wavelengths from three fixed wavelengths: 530,520 , and $510 \mathrm{~nm}$ for both KTM (left-hand panel) and JJK (right-hand panel). The position of the vertical arrows indicates the division between the two spectral sensations, which are green and blue in appearance.

variation in the fixed wavelength, the divisions between the two colors show a variation in their location of $2-3 \mathrm{~nm}$.

Overall, the two subjects have produced very similar results that are consistent with the three boundaries that divide the four colors, having constant spectral locations to within $3 \mathrm{~nm}$.

\section{Test for a Rod Contribution to Discrimination}

One question that arises concerns the possible contribution of rods to the detection and discrimination of the test spots. Such a contribution is unlikely for a number of reasons. First, the stimulus falls effectively within the rod-free area (1.7-2.0 deg), although it remains conceivable that eye movements might make a rod contribution possible. Second, at the background field intensity of $1000 \mathrm{Td}$, rods are substantially, although not completely, saturated ${ }^{21,22}$ and so are unlikely to contribute to detection thresholds. However, as an additional control, some of the detection and discrimination experiments were repeated with a more intense background (10,000 Td), which is well above rod saturation, and with a less intense background of $100 \mathrm{Td}$. Only the detection and discrimination of the shorter wavelengths were repeated under these conditions, since any rod contribution will be greatest at these wavelengths. Results are shown in Fig. 8.

Discrimination of shorter wavelengths from $520 \mathrm{~nm}$ (blue from green), to which rods are most likely to contribute, is unaffected by the higher background intensity. Furthermore, the imperfect discrimination between blue and violet found at the background of $1000 \mathrm{Td}$ also fails to disappear at the background of $10,000 \mathrm{Td}$. At the lower background illuminance of $100 \mathrm{Td}$, the blue/violet discrimination appears to be slightly improved. These results confirm that rods do not contribute to these color sensations identified at threshold.

\section{Spectral Sensitivity}

An outstanding question concerns the nature of the neural mechanisms subserving these different spectral color sensations. Are they postreceptoral in origin, and, if so, what is their link with the responses of the cones? Information concerning the identity of the mechanisms subserving the detection of the stimuli is available from the spectral sensitivity functions for the test stimuli.

Spectral sensitivity functions for the spot stimuli are shown for both subjects in Fig. 9. The functions were measured by using a method of adjustment to set the detection thresholds for the stimuli, which has the advantage of allowing the results to be collected at one sitting, rather than using the existing detection data obtained from many separate forced choice experiments. The vertical arrows mark the boundaries among the different color sensations and represent the averaged boundary locations, taken from the previous experiments. A characteristic triple-peaked sensitivity function is obtained, and the boundaries dividing the separate color sensations correspond to the divisions between the peaks and the troughs of the function. This shows that the M- and L-wavelength peaks of the function are each associated with a single color sensation (green and orange, respectively). A separable sensation (pale yellow) is associated with wavelengths located in the troughs between the M- and L-wavelength peaks. Finally, there is evidence that the short-wavelength peak may not be unitary under these conditions. It is associated with the sensation of blue at threshold, although at the shorter wavelengths a less distinct sensation of violet intrudes.

\section{DISCUSSION}

First, we have found that there are at least four perfectly distinguishable color sensations across the spectrum at threshold levels of detection. This suggests that the detection of the spectrum at threshold is subserved by at least four types of broadly tuned neural mechanism, each associated with a different color sensation. The boundaries between these mechanisms are characterized by sharp changes in discrimination. Second, the data show that these mechanisms have relatively fixed spectral locations, since the location of the different mechanisms is insensitive to the wavelength of the fixed stimulus in the discrimination. The 


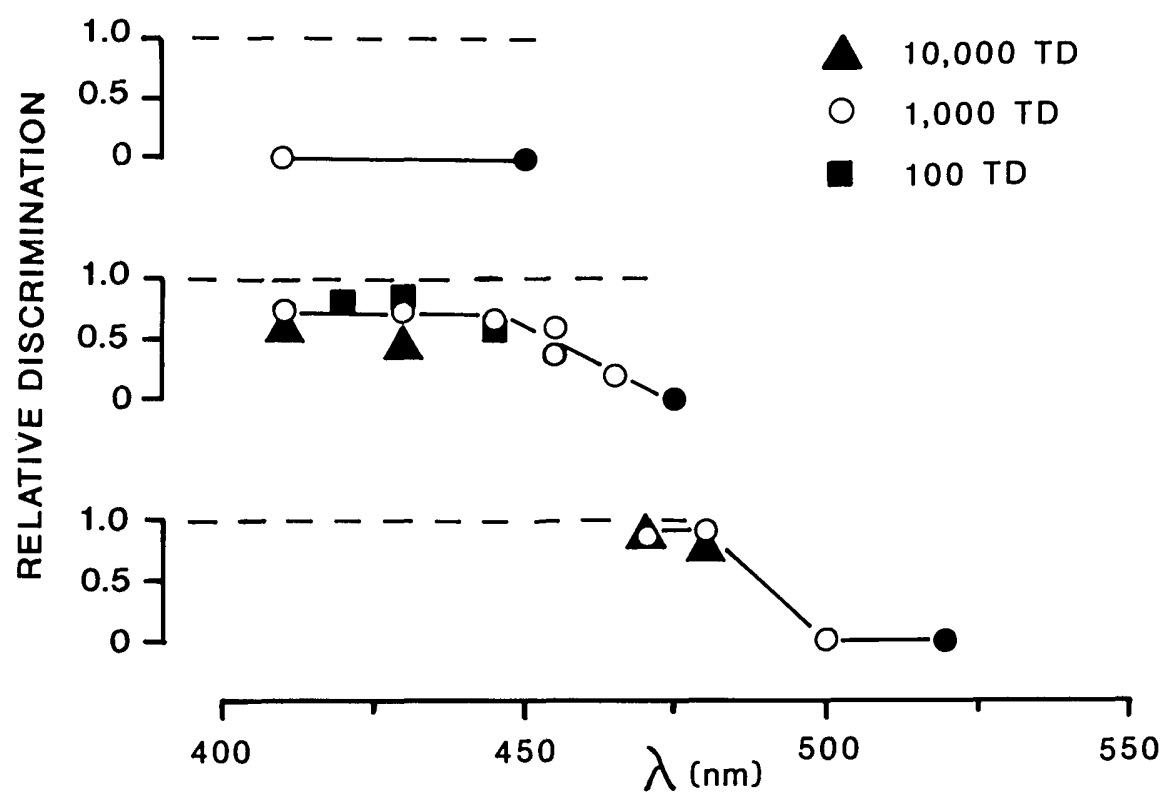

Fig. 8. Relative discrimination at detection threshold is plotted as for Figs. 3-7. Open circles show the results for discrimination of the three short-wavelength spectral sensations, green, blue, and violet, taken from Fig. 3. Triangles show results for discriminations from the same fixed wavelengths with a white background a log unit brighter $(10,000 \mathrm{Td})$, and squares show results for a white background a log unit dimmer (100 Td). (Subject KTM.)

results suggest that discrimination requires that the two stimuli lie on either side of a fixed perceptual boundary in the spectrum.

\section{Neural Basis of Threshold Detection Mechanisms}

The correspondence of the different spectral sensations with the peaks or the troughs of the triple-peaked spectral sensitivity function is indicative of their physiological basis. It is now generally accepted that the middle- and long-wavelength peaks of this spectral sensitivity function are both the wrong shape and in the wrong spectral locations to arise from the activities of a single-cone type. Instead, detection is determined by postreceptoral mechanisms with the middle- and long-wavelength peaks arising from cone opponent interactions. ${ }^{19,23,24}$

The present results show that these middle- and longwavelength peaks are each associated with a single color sensation (green and orange, respectively). Hence each peak is likely to arise from the output of a different type of broadly tuned mechanism rather than representing the upper envelope of detection by more numerous mechanisms, more narrowly tuned to a wavelength that would provide many more distinguishable colors across the spectrum. The spectral sensation of green is likely to arise from the output of a cone opponent channel combining M-cone responses with L-cone inhibitions (M-L), and the spectral sensation of orange is likely to arise from a (L-M) cone opponent response. Thus these color sensations appear to be determined by postreceptoral mechanisms, and discrimination in these two spectral regions is subserved by different broadly tuned mechanisms originating from the two types of cone opponent arrangements, (M-L) and (L-M).

The results also show that a separate neural mechanism determines detection in the trough between the middle- and long-wavelength peaks. It is likely that this is formed by some combination of $\mathrm{M}$ and $\mathrm{L}$ cones. However, this might occur as part of a luminance mechanism, in which case at detection threshold the subject would be making an intensity discrimination of the test spot from the background. Alternatively, this mechanism might form part of a cone opponent response in combination with short-wavelength (S) cones. There is little evidence by which to distinguish these two possibilities. On one hand, subadditivity at threshold has been shown to occur between wavelengths in the trough of the spectral sensitivity function and wavelengths from the $\mathrm{S}$ peak, ${ }^{24}$ suggesting the presence of weak cone opponent processes. On the other hand, suprathreshold wavelength discrimination in this spectral region is not influened by modulation of the $\mathrm{S}$ cones, suggesting the absence of S-cone opponent influences. ${ }^{25}$ It is likely that the potential contribution of either type of mechanism in this narrow region depends on the exact stimulus conditions and background illumination used.

Especially in one of the two subjects, there is evidence for a fifth poorly discriminated violet sensation at short wavelengths. Our informal experiments (not shown) indicate that the two ends of the spectrum can be distinguished apart at threshold, suggesting that the neural mechanisms subserving the detection of the shortest and the longest visible wavelengths are different. Furthermore, other distinguishable colors may exist in the nonspectral loci between the short and long wavelengths. A perceived redness of suprathreshold stimuli at short wavelengths is well known and may correspond to the threshold sensation that we labeled violet. However, there is no agreement on which receptors or postreceptoral arrangements are responsible for the short wavelength redness. Both an S-cone contribution to an $\mathrm{L}$ $\mathrm{M}$ cone opponent mechanism, ${ }^{26,27}$ and the involvement of secondary absorptions of short wavelengths by $\mathrm{L}$ cones have been proposed. ${ }^{28}$

It is worthwhile to compare these results, which reveal at least four different color sensations, with measurements ob- 
tained from the nonspectral color space. ${ }^{11}$ Discrimination at detection threshold was obtained among eight different, preselected axes in the color space. This suggests the presence of at least eight, higher-order mechanisms involved in detection. However, the positions of the dividing boundaries and the bandwidths of these mechanisms are not known. These differences cannot simply be explained by the presence of additional mechansims in the nonspectral loci $(400-700 \mathrm{~nm})$, and it remains unresolved why one approach should reveal higher-order mechanisms and the other lower-order ones.
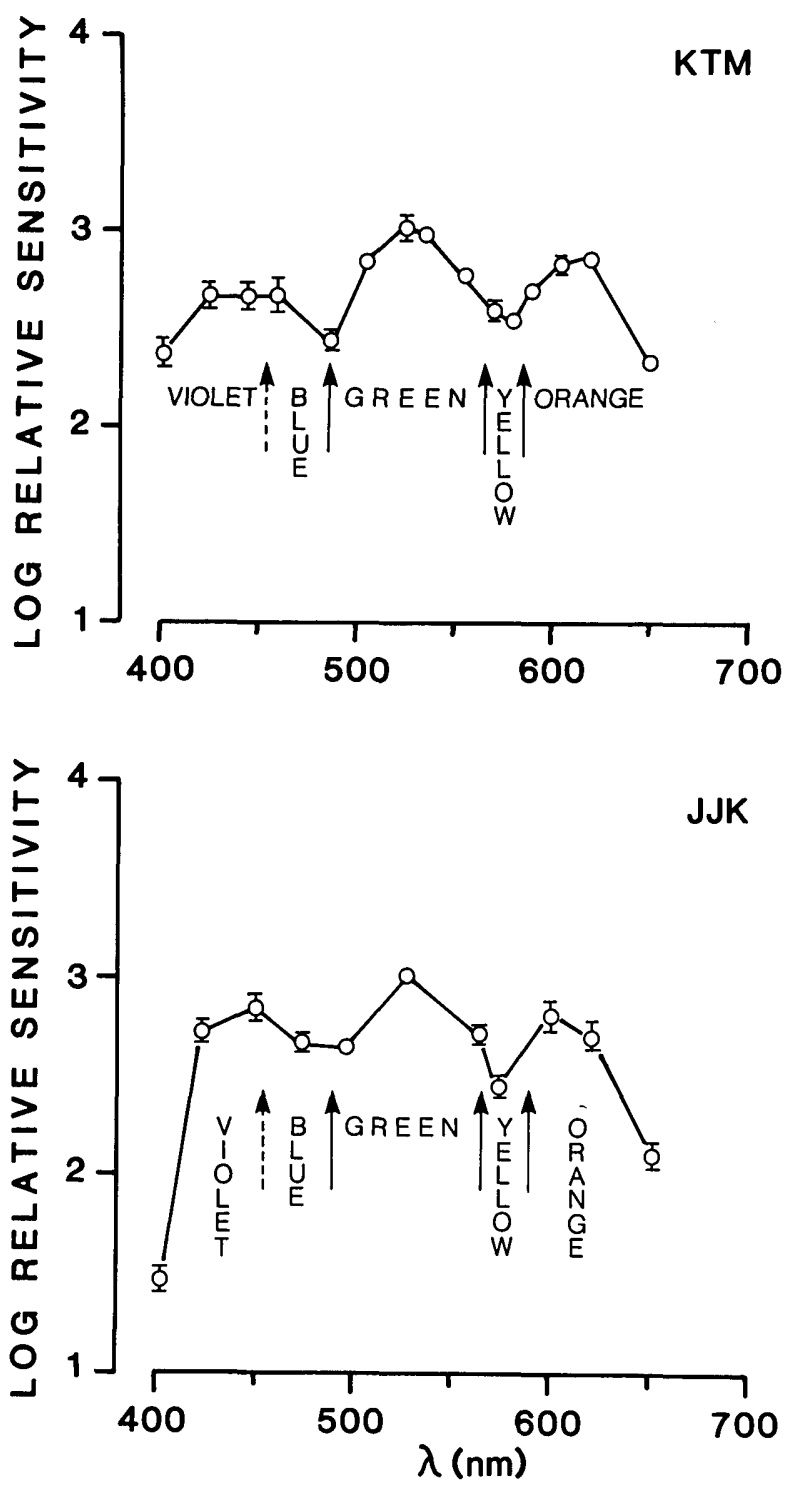

Fig. 9. Relative sensitivity to the test stimulus in $\log$ units as a function of the wavelength of the stimulus. Sensitivity is the reciprocal of the threshold intensity of the stimulus. Data points show the mean of four threshold settings with error bars of \pm 1 standard error. Arrows indicate the wavelength at the division between distinguishable spectral regions and show the averaged position taken from the previous figures. The dashed arrow indicates that discrimination between these two spectral regions is poor compared with that for the other three. The color of the spectral regions is indicated. Results are for KTM (top panel) and JJK (bottom panel).
Presence of Fixed Perceptual Boundaries in Spectrum and Categorical Limits on Discrimination

It was pointed out in the Introduction that the perfect discrimination of two stimuli at threshold levels of detection does not necessarily require an explanation in terms of a different type of labeled detector responding to each distinguishable stimulus, as in a threshold model. Alternatively, continuous models propose that discrimination depends on a change in the overall activity of arrays of tuned detectors, depending on their bandwidths and sampling density. These two types of model diverge in their interpretation of whether the boundaries between the distinguishable colors in the spectrum are likely to be fixed. If the boundaries dividing the discriminable colors in the spectrum occur at fixed spectral locations, and discrimination requires only that stimuli lie on either side of this perceptual boundary, then a discrete or categorical coding of the spectrum is implied. The threshold model allows for a discrete neural encoding at threshold and so can readily account for the presence of fixed perceptual boundaries between chromatic stimuli and chance levels of discrimination within a categorical sensation.

Continuous models would have more difficulty in accounting for fixed perceptual boundaries. For example, this type of model would predict that, as the fixed wavelengths in the midspectral region are moved toward the green-yellow boundary and hence toward the more narrowly tuned detectors found in the yellow region, there would be a steepening in relative discrimination as a function of wavelength and an associated shift in border location. This is not evident in the results, which show that the transitions from the green to yellow regions are of almost identical shape and location regardless of the fixed wavelength (Fig. 5). A similar relationship is seen for the borders dividing the yellow and orange distinguishable colors (Fig. 6). The presence of these invariant perceptual boundaries in the spectrum implies that discrimination in these spectral regions has a categorical basis. Categorical performance limits on red-green discriminations of flickering test stimuli have been reported previously and are thought to arise from the contribution of a luminance mechanism to the task. ${ }^{29}$ If the yellow mechanism is in fact a luminance mechanism, the present results would support those of Wandell ${ }^{29}$ in suggesting that categorical boundaries exist between luminance and color opponent mechanisms.

The border between green and blue also shows no more than 3-nm variation in position (Fig. 7). However, there is some suggestion in the data that this border is slightly less rigid in shape than the other two, with a slight steepening of the discrimination function as the fixed wavelength is moved nearer the border. Such effects might be caused by probability summation occurring between different mechanisms, and in this limiting case it becomes difficult to distinguish between this or other causes. Further experiments, for example along the lines of Gille ${ }^{30}$ and Rollman and Nachmias, ${ }^{7}$ may be useful for determining which model is more appropriate for describing detection and discrimination in each spectral region. However, we emphasize that the chosen method of analysis does not influence the actual results obtained. We reanalyzed all the results for subject KTM using the method of Thomas and Gille, ${ }^{3}$ and this revealed boundaries with an overall greater variability but of similar 
shape and in the same spectral locations (to within $1.5 \mathrm{~nm}$ ) as those already described in the figures.

A categorical basis for color sensation has been proposed from a study of color naming in which, excluding black, white, and gray, eight basic color names were found..$^{31,32}$ However, despite the coincidence in the use of color names between the two studies, it is unclear whether the sensations to which the names refer also correspond. At suprathreshold intensities, the four spectral mechanisms identified at threshold are likely to be combined into higher-order mechanisms, potentially producing a different range of sensations. Thus only a study of the color naming of threshold stimuli would allow more direct comparisons to be drawn.

\section{ACKNOWLEDGMENTS}

We are grateful to $\mathrm{D}$. Carden for his invaluable assistance during these experiments. We are also grateful to $\mathrm{H}$. B. Barlow for helpful discussions and to J. D. Mollon, J. Krauskopf, and M. Webster for helpful comments on the manuscript. The support of the Vision Research Trust is acknowledged. The experiments were done at the Department of Ophthalmic Optics, University of Manchester Institute of Science and Technology, Manchester, UK.

\section{REFERENCES}

1. J. Nachmias and A. Weber, "Discrimination of simple and complex gratings," Vision Res. 15, 217-223 (1975).

2. D. J. Tolhurst and R. S. Dealy, "The detection and identification of lines and edges," J. Opt. Soc. Am. 69, 652-660 (1975).

3. J.P. Thomas and J. Gillie, "Bandwidths of orientation channels in human vision," J. Opt. Soc. Am. 69, 652-660 (1979).

4. A. B. Watson and J. G. Robson, "Discrimination at thresholds: labelled detectors in human vision," Vision Res. 21, 1115-1122 (1981).

5. J. P. Thomas, "Detection and identification: how are they related?” J. Opt. Soc. Am. A 2, 1457-1467 (1985).

6. R.F. Hess and G. T. Plant, "Temporal frequency discrimination in human vision: evidence for an additional mechanism in the low spatial and high frequency region," Vision Res. 25, 14931500 (1985).

7. G. B. Rollman and J. Nachmias, "Simultaneous detection and recognition of chromatic flashes," Percept. Psychophys. 12, 309-314 (1972).

8. B. A. Wandell, J. Sanchez, and B. Quinn, "Detection/discrimination in the long-wavelength pathways," Vision Res. 22, 10611069 (1982).

9. D. B. Kirk, "Color discrimination at threshold: the approach through incremental threshold sensitivity," Vision Res. 22, 713720 (1982).

10. D. C. Hood and M. A. Finklestein, "A case for the revision of text book models of colour vision: the detection and appearance of small brief lights," in Colour Vision: Physiology and Psychophysics, J. D. Mollon and L. T. Sharpe, eds. (Academic, London, 1983), pp. 385-398.

11. J. Krauskopf, D. R. Williams, H. B. Mandler, and A. M. Brown, "Higher order color mechanisms," Vision Res. 26, 23-32 (1986).
12. S. Zeki, "The representation of colours in the cerebral cortex," Nature (London) 284, 412-418 (1980).

13. J. Krauskopf and R. Srebro, "Spectral sensitivity of color mechanisms: derivation from fluctuation of color appearance near threshold," Science 150, 1477-1479 (1965).

14. J. Krauskopf, "On identifying detectors," in Visual Psychophysics and Physiology, J. C. Armington, J. Krauskopf, and B. R. Wooten, eds. (Academic, New York, 1978), pp. 283-295.

15. P. E. King-Smith and D. Carden, "Luminance and opponent colour contributions to visual detection and adaptation, and to temporal and spatial integration," J. Opt. Soc. Am. 66, 709-717 (1976).

16. D. H. Foster and R. S. Snelgar, "Test and field spectral sensitivities of colour mechanisms obtained on small white backgrounds: action of unitary opponent colour processes?" Vision Res. 23, 787-797 (1983).

17. E. M. Granger and J. C. Heurtley, "Visual chromaticity-modulation transfer function,” J. Opt. Soc. Am. 63, 1173-1174 (1973).

18. K. T. Mullen, "The contrast sensitivity of human colour vision to red-green and blue-yellow chromatic gratings," J. Physiol. 359, 381-409 (1985).

19. H. G. Sperling and R. J. Harwerth, "Red-green cone interactions in increment threshold of spectral sensitivity of primates," Science 172, 180-184 (1971).

20. K. T. Mullen, J. J. Kulikowski, and D. Carden, "The identification of spectral colour sensations," in Seeing Contour and Colour, J. J. Kulikowski and C. M. Dickenson, eds. (Pergamon, London, 1989), pp. 261-266.

21. M. Aguilar and W. S. Stiles, "Saturation of the rod mechanism of the retina at high intensity levels of stimulation," Opt. Acta 1, 59-65 (1954).

22. R. F. Hess and K. Nordby, "Spatial and temporal limits of vision in the achromat," J. Physiol. 371, 365-385 (1986).

23. K. Kranda and P. E. King-Smith, "Detection of coloured stimuli by independent linear systems," Vision Res. 19, 733-745 (1979).

24. J. E. Thornton and E. N. Pugh, Jr., "Relationship of opponentcolours cancellation measures to cone-antagonistic signals deduced from incremental threshold data," in Colour Vision: Physiology and Psychophysics, J. D. Mollon and L. T. Sharpe, eds. (Academic, London, 1983), pp. 361-373.

25. J. D. Mollon and C. R. Cavonius, "The chromatic antagonisms of opponent process theory are not the same as those revealed in studies of detection and discrimination," in Colour Vision Deficiencies VII, G. Verriest, ed. (Junk, The Netherlands, 1987), pp. 473-483.

26. D. Jameson and L. M. Hurvich, "Opponent response functions related to measured cone photopigments," J. Opt. Soc. Am. 58, 429-430 (1968).

27. B. R. Wooten and J. S. Werner, "Short-wave cone input to the red-green opponent channel," Vision Res. 19, 1053-1054 (1979).

28. F. M. De Monasterio and P. Gouras, "Responses of macaque ganglion cells to far violet lights," Vision Res. 17, 1147-1156 (1977).

29. B. A. Wandell, "Color measurement and discrimination," J. Opt. Soc. Am. A 2, 62-71 (1985).

30. J. Gille, "Detection and identification of color increments to a white background," J. Opt. Soc. Am. A 1, 1241 (1984).

31. R. M. Boynton and C. X. Olson, "Locating basic colors in the OSA space," Color Res. Applic. 94 (1987).

32. K. Uckikawa and R. M. Boynton, "Categorical color perception of Japanese observers: comparison with that of Americans," Vision Res. 27, 1825-1833 (1987). 